\title{
Andamento della conducibilità elettrica dell'aria nella Regione Adriatica Centrale
}

\author{
(Behavior of the electrical conductivity of the air \\ in the Central Adriatic Region)
}

\author{
A. Murri - R. Fusari - C. Scuterini $(*)$
}

Ricevuto il 10 Maggio 1974

Rinssunto. - Durante il periodo Ottobre 1970/Marzo 1974, sono state compiute misure continue di conducibilità dell'aria e ricerche sul pulviscolo atmosferico. Della conducibilità dell'aria sono stati calcolati gli andamenti medi stagionali ed annui, e ne sono stati esaminati i comportamenti durante fenomeni meteorologici caratteristici come temporali, nebbie, foehn.

Si sono infine confrontati gli andamenti annui medi della conducibilità totale e del pulviscolo atmosferico, notando che esiste un marcato anti. parallelismo dei due fenomeni.

Sumary. - Smoothed hourly averages of electrical conductivity of the air, are calculated, on the basis of continuous recording wich are carried out in the central adriatic region, using Gerdien cylindrical condensers, during the years $1970 / 1974$.

Dissimilarities between thundersthorms, fogs and foehn winds are evaluated, together with the hourly behavior of the dust, which result greatly antiparallel to the behavior of the electrical conductivity of the air.

1. - PREMESSA

L'Osservatorio Geofisico e Meteorologico di Macerata $\left(43^{\circ} 17^{\prime}+3^{\prime \prime}\right.$ $\mathrm{N}-13^{\circ} 27^{\prime} 08^{\prime \prime} \mathrm{EG}-338$ m.s.l.m.) ha iniziato nell'ottobre del 1970

(*) Osservatorio Geofisico di Macerata. 
misure di conducibilità elettrica dell'aria, dapprima nella polarità negativa e poco ropo anche nella polarità positiva.

Gli strumenti alimentati in C.C., sono stati protetti con diversi accorgimenti dall'umidità, allo scopo di evitare per quanto possibile le conseguenti dispersioni.

Grande cura è stata posta nella costante pulizia della parte soggetta al flusso d'aria, onde evitare perdite a causa dei repositi di pulviscolo atmosferico, particolarmente notevoli in inverno e clurante la presenza delle sabbie portate rlai venti di $\mathrm{S}$ e $\mathrm{SW}$, in coincidenza di caratteristiche situazioni di tempo in grande.

Le misure durante la campionatura ed in seguito, sono state sempre compiute contemporaneamente a misure di grarliente di potenziale, eseguite con elettrometro a valvola e controllate mediante elettrometro campione di Keitley.

Le misure sistematiche, condlotte in modo continuo nelle 24 ore e per tutto il tempo, sono iniziate subito dopo aver terminato l'accurato campionamento degli strumenti.

I risultati medi esposti si riferiscono a giornate elettricamente calme e totalmente serene. Inoltre sono riportati i risultati ottenuti in casi particolari, come giornate con nebbia, foehn, etc.

Delle giornate elettricamente calme ne sono state scelte cinque con metorli probabilistici, onde ricavare i valori merli.

Le giornate coincidono con quelle scelte per ricavare $i$ valori merli rlel gradiente di potenziale.

\section{2. - ANDAMENTO ORARIO DELLA CONDUCIBILITÀ}

Gli andamenti orari della conducibilità per giornate serene ed elettricamente calme, sono quelli inclicati dalle figg. 1 e 2 .

Abbiamo ripartito gli andamenti stessi per stagioni meteorologiche in morlo da mettere in evidenza le eventuali influenze stagionali e locali. La fig. 2 indica l'andamento medio orario annuo.

Come si rileva dal grafico, le curve stagionali presentano diversità assai accentuate e caratteristiche comuni.

Inverno. L'andamento da noi trovato per il periodo considerato, appare in armonia con quanto ottenuto da altri ricercatori per stazioni europee ed extraeuropee $(1,2,5,8,10,12,18)$. 
Esso presenta caratteristiche continentali e marittime ad un tempo, data la posizione della stazione a non grande distanza dal mare Adriatico ed a breve distanza dalla parte più elevata della catena appenninica.

Da notare il caratteristico massimo nei dintorni delle ore 4.00 locali, in coincidenza con il minimo di potenziale, ed un minimo fra le ore 17 e le ore 18 locali, che corrisponde anche esso ad un massimo stagionale del gradiente di potenziale, già da noi messo in rilievo ( $\left.{ }^{13}\right)$. Il resto dell'andamento invernale non presenta altre particolaritì notevoli, almeno nelle giornate elettricamente calme, salvo il piccolo minimo fra le ore 2 e le 3 locali, minimo che si ripresenterà in tutti gli andamenti stagionali con qualche spostamento dell'ora, e che si ritrova abbastanza ben definito anche nell'andamento medio e orario annuo.

Primavera. L'andamento primaverile è quello che appare più accentuato nei suoi valori, in relazione all'influenza che $i$ venti dominanti della stagione hanno sulla conducibilità dell'aria, come pure le brezze che si determinano localmente.

Caratteristici dell'andamento primaverile sono il minimo e il massimo molto accentuati che si hanno verso le ore 6.00 e le 20.00 locali. L'andamento primaverile presenta anche esso un piccolo minimo verso le ore 3.00, già notato nell'andamento invernale, e che non abbiamo riscontrato in rilievi eseguiti in altre stazioni.

Tutto l'andamento relativo ai mesi di Marzo, Aprile, e Maggio appare assai mosso, rispecchiando le diverse situazioni meteorologiche che si determinano e si succedono in tali mesi di transizione, influenzando anche le giornate più calme.

Estate. L'andamento in questa stagione presenta, oltre al solito massimo delle ore 4.00 locali, l'accentuato minimo dovuto all'effetto della radiazione solare nelle prime ore del mattino, fino al momento in cui si determinano in modo massiccio i fenomeni di rimescolamento degli strati.

Il resto dell'andamento appare regolarmente crescente con il progredire delle ore notturne, durante le quali nei mesi estivi, localmente, si ha prevalenza di calma di vento, contrariamente a quanto avviene durante i mesi primaverili.

A utunno. I valori autumnali della conducibilità si presentano assai più alti di quelli delle precedenti stagioni e prossimi ai valori invernali, che risultano i più elevati di tutto l'anno. 
Anche in autunno l'andamento della conducibilità presenta il piccolo minimo fra le ore 2.00 e le ore 3.00 locali, immerliatamente seguito dal massimo ben noto delle ore 4.00 , in concomitanza, come è stato già detto, del minimo di gradiente verticale di potenziale.

Il resto dell'andamento orario si presenta normale, e simile a quello rlei mesi invernali.

Andamento generale. iे quello indicato in fig. 2 e nella tabella 1. isso presenta il massimo alle ore 4.00 locali, ed un accentuato minimo nelle ore centrali della giornata, in corrispondenza del massimo di gradiente. Quindi i valori della conducibilità aumentano nelle ore pomerirliane e notturne.

I valori assunti dalla polaritì negativa sono leggermente superiori a quelli assunti dalla polarità positiva, come è facilmente rilevabile dalla tavola. Tale anclamento è concordante con quanto rilevato dalla maggioranza dei ricercatori che hanno trattato il problema.

Qualche inversione in tale andamento, con valori della polariti positiva superiori cioè a quelli della polarità negativa, si sono rilevati in rare occasioni. Nell'andamento diurno merlio annuo è notevole il fatto che i valori delle due polarita si avvicinino molto, fino ad irlentificarsi verso le ore 15.00 di T.M.F.C.

I'andamento generale presenta, come quelli stagionali, un piccolo minimo alle ore 2.00 circa. s vir.. tale minimo, come gir si è detto, non compare negli andamenti trovati per altre su--joni, abbiamo ritenuto di inclagare se esso potesse essere comnesso con fenomeni locali, lata la sua persistenza in tutte le serie di misure eseguite nelle quattro stagioni.

Poiché si è notato che al momento in cui si determina un cambiamento di direzione o di intensità del vento, oppure si passa da un periodo di calma ad un periodo di vento, la conducibilità atmosferica dei due segni subisce una variazione, si è indagato mediante una ricerca statistica sulle possibili cause di formazione del piccolo minimo nei dintorni delle ore 2.00 locali.

Si ì pertanto esaminato l'andamento della direzione del vento nelle ore comprese fra le ore 0.00 di ciascun giorno elettricamente calmo, usato per calcolare le medie della conducibilità, e le ore 4.00 . I risultati di tale indagine statistica hanno messo in evidenza che il piccolo minimo della conducibilità dell'aria verso le ore 2.00 locali, puó ragionevolmente connettersi con la prevalenza in quelle ore di venti locali provenienti dal settore $\mathrm{SW}-\mathrm{W}-\mathrm{NW}$. 


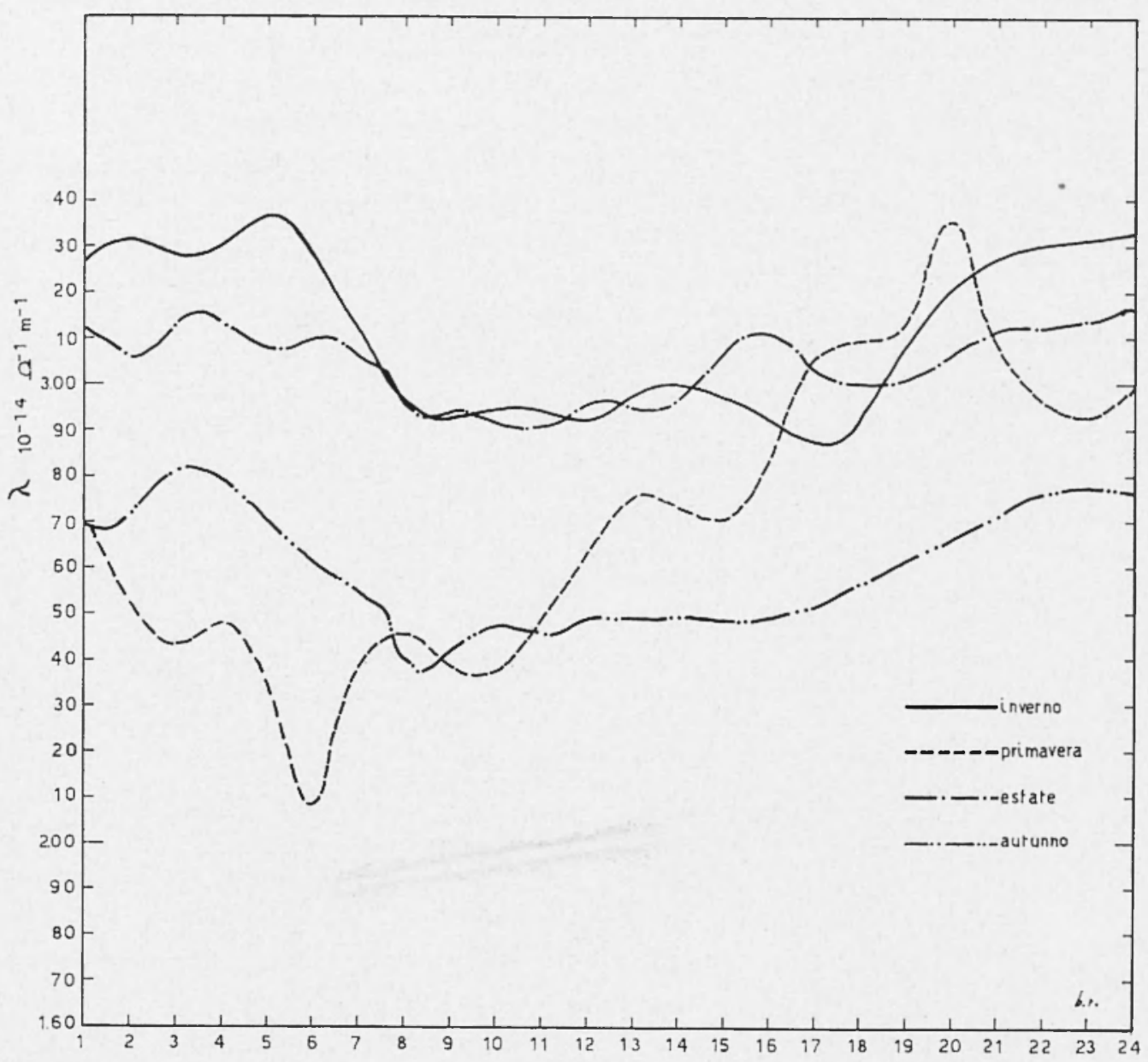

Fig. 1. - Andamento stagionale della conducibilità dell'aria, I valori medi sono ricavati dalle osservazioni del periodo 1970-1974 (Marzo).

Tali direzioni indicano provenienza dall'interno della regione, ed in particolare dal massiccio appenninico.

La formazione di venti di questo genere si è constatata nell'82\% dei casi esaminati, che in particolare cadono con la frequenza maggiore nelle stagioni intermedie: primavera ed autunno.

Durante tali stagioni l'efletto appare assai accentuato, come è ben riscontrabile dai relativi grafici della fig. 1. 


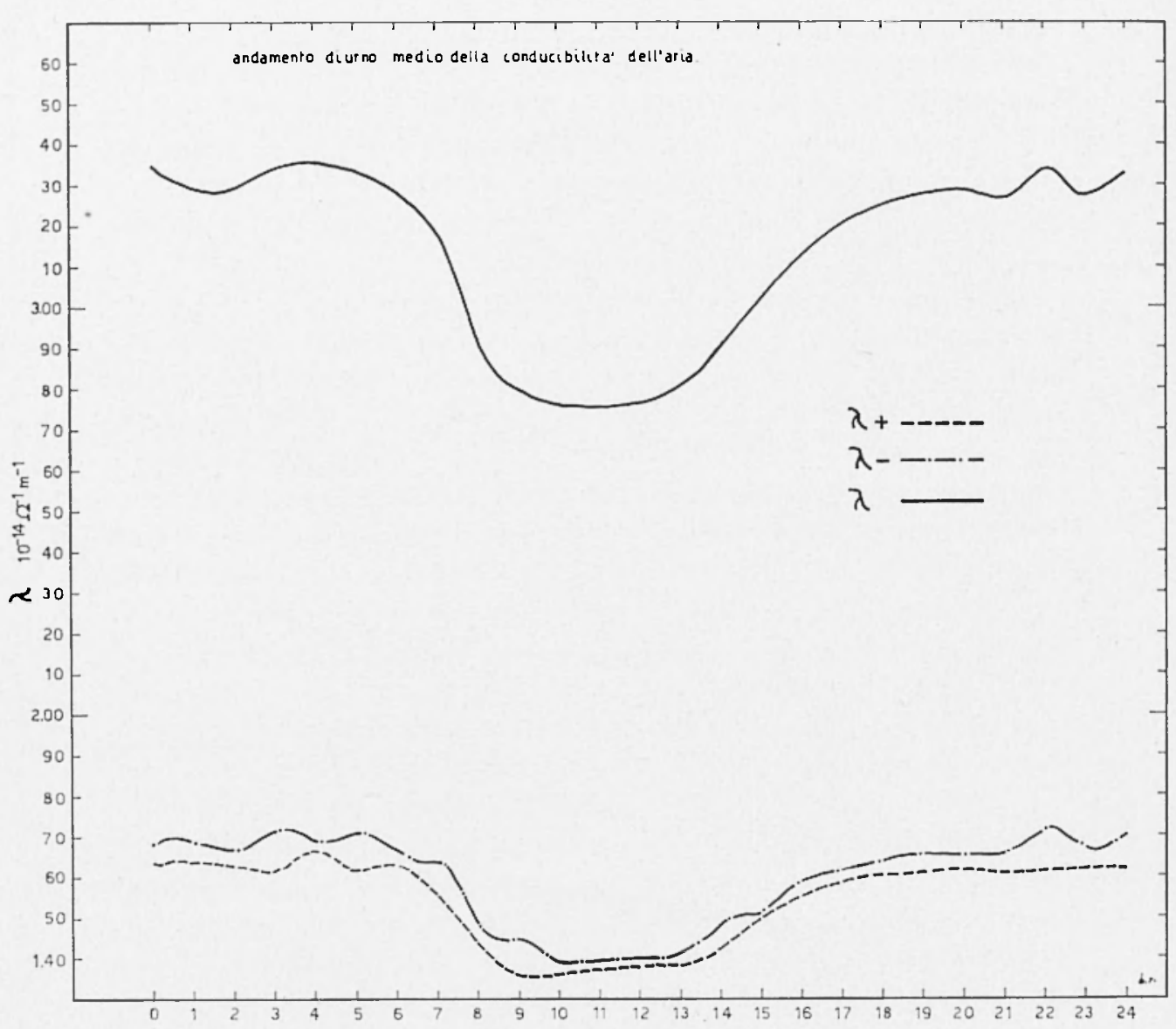

Fig. 2. - Andamento della conducibilità elettrica dell'aria, durante la gior. nata. Le curve inferiori indieano rispettivamente l'andamento della polarita positiva e di quella negativa. Valori tratti dalle osservazioni del periodo $1970-74$ (Marzo)

Nelle stagioni invernale ed estiva la frequenza dei venti dal quadrante $\mathrm{W}$, alle ore considerate, è assai minore e l'effetto riscontrato appare di minore intensità. Occorre aggiungere che nella stessa inclagine è stato messo in luce che per il restante $18 \%$ dei casi, durante $\mathrm{i}$ quali non si sono avuti venti dal quadrante occidentale, si sono verificati - in occasione di venti da NE, E, SE - aumenti della conducibiliti dell'aria.

L'effetto d'altra parte può apparire perfettamente normale se si considera che i venti dal quadrante $\mathrm{E}$, nella regione, giungono alla 
stazione di osservazione dopo aver attraversato il mare Adriatico nella sua maggiore ampiezza. La provenienza del vento pertanto giustifica l'aumento della conducibilità, in quanto è stato rilevato da tutte le osservazioni, come la conducibilità dell'aria sul mare sia maggiore che non sulla terra $(1,4,9,19)$.

In particolare poi è da notare che il minimo in vicinanza delle ore 2.00 locali cade per le due polarità, in ore diverse.

Quello della polarita positiva si determina circa mezz'ora prima di quello della polarità negativa, come si puó rilevare dalla fig. 2.

TABella 1

\begin{tabular}{|c|c|c|c|}
\hline Ora & $\lambda_{-}$ & $\lambda_{+}$ & $\lambda$ \\
\hline 1 & 1.69 & 1.64 & 3.33 \\
\hline 2 & 1.67 & 1.63 & 3.30 \\
\hline 3 & 1.72 & 1.62 & 3.34 \\
\hline 4 & 1.69 & 1.66 & 3.35 \\
\hline 5 & 1.71 & 1.62 & 3.33 \\
\hline 6 & 1.66 & 1.63 & 3.29 \\
\hline 7 & 1.64 & 1.56 & 3.20 \\
\hline 8 & 1.48 & 1.44 & 2.92 \\
\hline 9 & 1.45 & 1.35 & 2.80 \\
\hline 10 & 1.39 & 1.36 & 2.75 \\
\hline 11 & 1.38 & 1.37 & 2.74 \\
\hline 12 & 1.39 & 1.37 & 2.76 \\
\hline 13 & 1.42 & 1.38 & 2.80 \\
\hline 14 & 1.48 & 1.42 & 2.90 \\
\hline 15 & 1.50 & 1.50 & 3.00 \\
\hline 16 & 1.58 & 1.54 & 3.12 \\
\hline 17 & 1.62 & 1.58 & 3.20 \\
\hline 18 & 1.64 & 1.60 & 3.23 \\
\hline 19 & 1.66 & 1.60 & 3.26 \\
\hline 20 & 1.66 & 1.62 & 3.29 \\
\hline 21 & 1.66 & 1.60 & 3.26 \\
\hline 22 & 1.73 & 1.61 & 3.34 \\
\hline 23 & 1.66 & 1.62 & 3.28 \\
\hline 24 & 1.71 & 1.61 & 3.32 \\
\hline
\end{tabular}


3. - Comportimexto Della ConducibilitA ElétTrich DELl'ARia DLRANTE DIVERSI EVENTI METEOROLOGICI

Nebbia - Ia fig. 3 mostra la registrazione del comportamento della conducibilità (componente negativa) dell'aria durante l'improvviso formarsi di un banco di nebbia (3 Marzo 1973) nella notte, causato da un abbassamento della temperatura, per un brusco salto del vento da SE a NNW. Il salto del vento si effettua verso le ore 1.05 e ad esso corrisponde un primo picco nella registrazione della conducibilità, la quale ritorna per circa mezza ora ai valori primitivi. Al momento in cui la sonda viene a trovarsi immersa nel banco di nebbia formatosi, i valori della conducibilitì passano da $2,68 \cdot 10^{-14}\left(\mathrm{omh}^{-1} \mathrm{~m}^{-1}\right)$ a $3,80 \cdot 10^{-14}\left(\mathrm{omh}^{-1} \mathrm{~m}^{-1}\right)$ assumendo bruscamente un valore molto superiore a quello precedente.

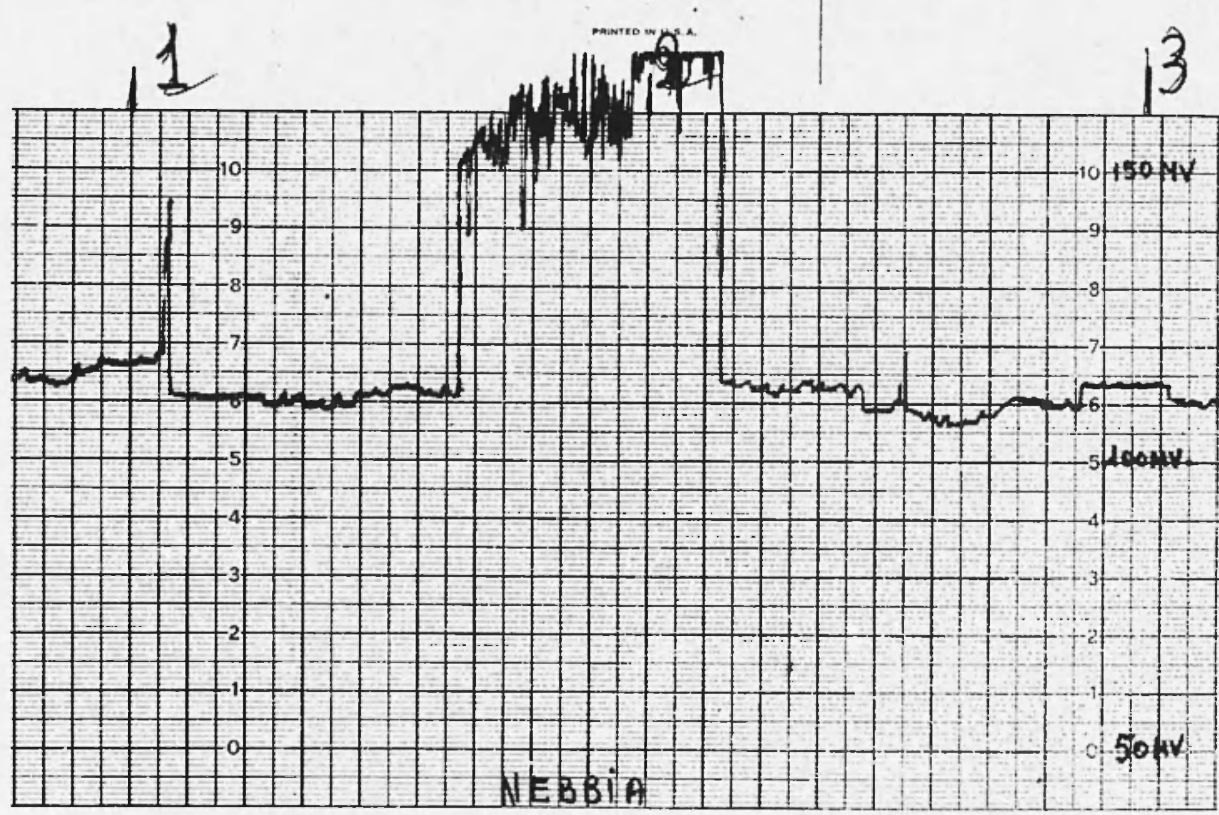

Fig. 3. - Andamento della conducibilità dell'aria (polarità negativa) durante un caso di nebbia. L'andamento della polarità positiva appare parallelo. 
Le oscillazioni della conducibilità attorno ad un valore medio appaiono assai accentuate, presentando notevoli picchi per valori superiori ed inferiori al valore medio di $3,30 \cdot 10^{-14}\left(\mathrm{omh}^{-1} \mathrm{~m}^{-1}\right)$.

Il ritorno al valore precedente di $2,68 \cdot 10^{-14}\left(\mathrm{omh}^{-1} \mathrm{~m}^{-1}\right)$ avviene al momento in cui il banco di nebbia presente viene dissolto dal vento che soffia nuovamente in direzione SE.

Le caratteristiche da noi documentate in questo caso, si presentano sensibilmente eguali in tutti i casi simili in presenza di nebbia, qualunque sia la causa della formazione di questa.

Vi è solo da aggiungere che, in genere, le modificazioni riscontrate nei valori della conducibilità, dovute a nebbie formatesi per irraggiamento, hanno avuto una durata maggiore, e che le variazioni nel valore percentuale della conducibilità appaiono minori rispetto a quelle verificate in nebbie di altra origine.

Nel caso che si è considerato, con nebbia formatasi per il passaggio di una piccola bolla di aria più fredda, avente caratteristiche termodinamiche e di provenienza diverse da quelle possedute dall'aria presente nella zona di osservazione, le modificazioni avvenute nella conducibilità ammontano al $42 \%$ del valore primitivo.

In altri casi di nebbie si sono notati aumenti del valore della conducibilità minori di quello riportato; in qualche raro caso si è determinata una diminuzione del valore della conducibilitia.

Gli andamenti della conducibilità dei due sensi appaiono sempre paralleli.

Temporale - La fig. 4 evidenzia il comportamento delle due componenti della conducibilità dell'aria durante un temporale proveniente da SW (6 Febbraio 1974), la cui fase massima si è avuta nella zona della stazione di osservazione fra le ore 23.30 del 5 e le ore 2.00 del 6 Febbraio, T.M.E.C.

L'andamento della $\lambda$ in tale occasione appare molto caratteristico. Le modificazioni massime che si notano nella conducibilità dei due segni si verificano al momento del passaggio della cella principale del temporale sulla stazione di osservazione.

Mentre il valore della conducibilità negativa è di $1,08 \cdot 10^{-14}$ $\left(o h^{-1} \mathrm{~m}^{-1}\right)$ all'inizio del passaggio della cella temporalesca e quello della conducibilità positiva è di $1,05 \cdot 10^{-14}$, i valori raggiunti al momento del passaggio sono rispettivamente di $1,36 \cdot 10^{-14}$ e di $1,12 \cdot 10^{-14}$. Essi scendono dopo il passaggio del fronte a valori prossimi a quello di inizio. 


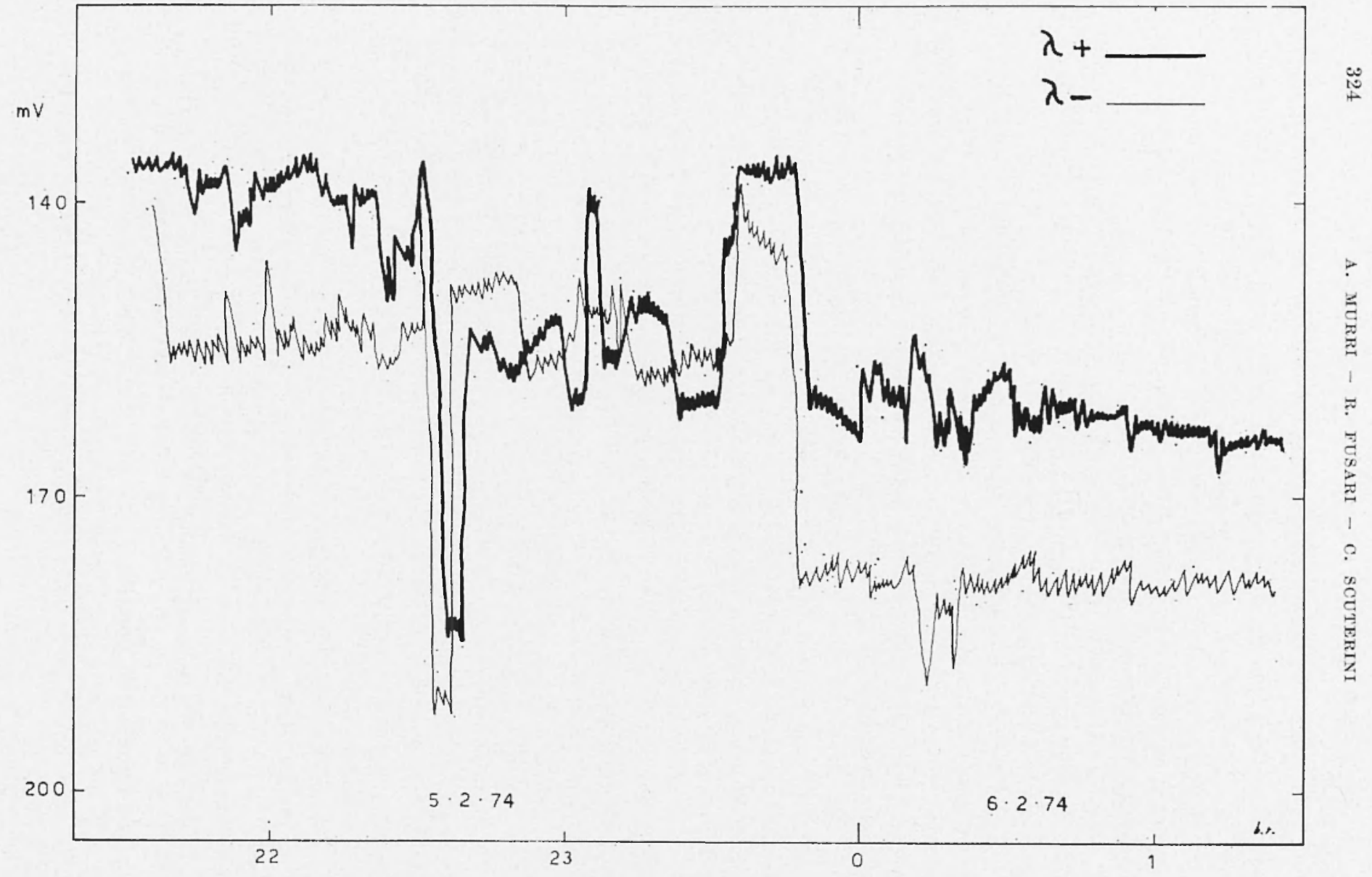

Fig. 4. - Andamento della conducibilità elettrica dell'aria durante un temporale. Sono messi in evidenza gli andamenti delle due polarità. 
E da notare ad ogni modo che le variazioni percentuali appaiono assai notevoli, per ambedue le polarita, anche se le variazioni della polarità positiva appaiono minori di quella negativa.

L'andamento rappresentato dalla fig. 4 è tipico per quasi tutti i temporali da noi registrati, e per tale ragione lo abbiamo prescelto. Circa due ore prima del manifestarsi del passaggio del fronte temporalesco i valori della conducibilità negativa tendono a crescere rispetto al valore del momento, presentando dei picchi particolari che si accentuano in frequenza el in valore all'avvicinarsi della cella temporalesca al luogo di osservazione.

Tali picchi corrispondono alle scariche isolate di forte intensità od a gruppi di scariche, e sono quasi certamente dovuti alla jonizzazione dell'aria attuata dal meccanismo della prescarica e scarica. Il passaggio del centro della cella sulla stazione o in sua prossimità determina un brusco, forte aumento della conducibilità dei due sensi, la quale dopo il passaggio della cella tende ad acquistare valori minori di quelli precedenti al formarsi ed al transito del temporale.

I valori tornano normali parecchie ore clopo. In genere si è notato che, mediamente, il periodo di tempo impiegato prima del temporale per passare da valori normali a valori anormali è circa la metà di quello necessario, dopo il temporale, a riportarsi al livello dei valori normali.

Foehn - La fig. 5 offre l'andamento della conducibilità (polarità positiva) mentre la tabella 2 indica i valori della conducibilità dei due segni durante un tipico evento di foehn da SW, verificatosi in periodo perturbato (6 Luglio 1973) fra le ore 11.00 e le ore 17.00 circa. Il fenomeno del foehn, che si determina nella regione nelle condizioni di tempo in grancle e con caratteristiche già a suo tempo studiate da uno di noi (13) determina uno specifico comportamento dei parametri elettrici dell'atmosfera.

Si può clire che, se il foehn forzato determina un aumento notevolissimo del gradiente di potenziale, portandolo a valori che sono assai superiori alla norma, il foehn libero determina invece delle oscillazioni del gradiente attorno al valore medio della giomata in cui il fenomeno si determina.

Oscillazioni simili sono state notate nella conducibilità dell'aria, come è specificatamente messo in luce dalla fig. $\breve{5}$ che riproduce il diagramma della polarità positiva rilevato durante una manifestazione di vento catabatico libero. 


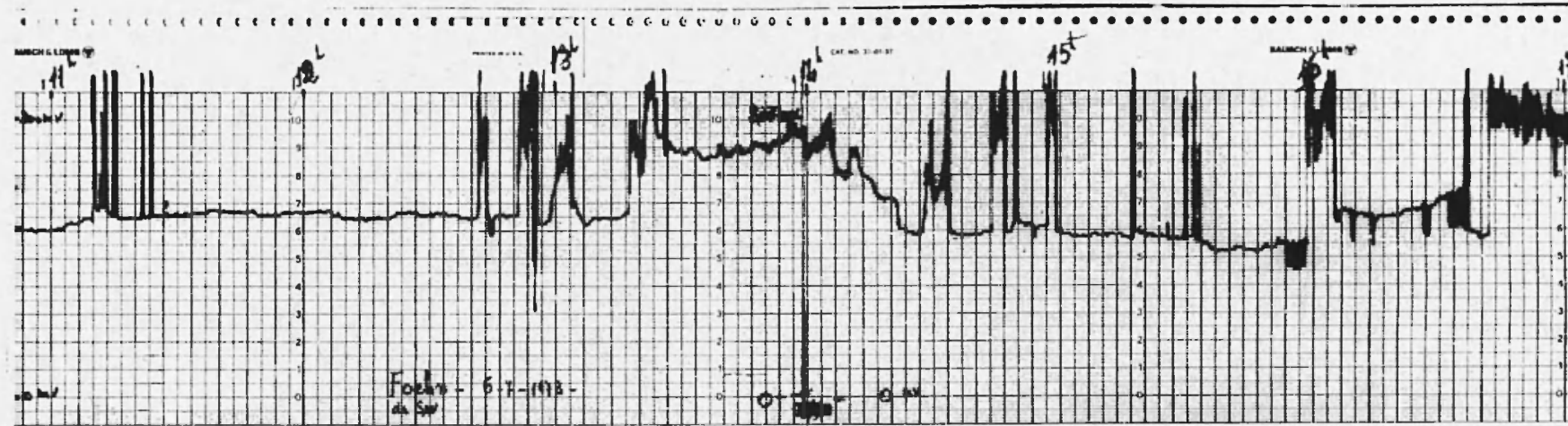

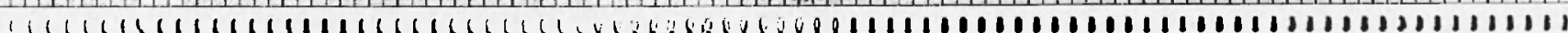

Fig. 5. - Andamento della conducibilità dell'aria (polarità positiva) durante un periodo di Foehn libero. L'andamento della polarità negativa appare parallelo. 
Le oscillazioni della conducibilitì sono state da noi tabellate, riportando i valori ogni 30 minuti, assieme alle corrispondenti variazioni del gradiente di potenziale.

Tali variazioni, d'altra parte molto caratteristiche, sono legate alle raffiche del vento, in corrispondenza delle quali si sono avute variazioni ed oscillazioni della conducibilità molto rapide, e tali da determinare dei picchi nei due sensi, con prevalenza di quelli tendenti ad aumentare il valore della conducibilità, fino a farle raggiungere valori che sono da considerarsi eccezionalmente alti, e caratteristici rel fenomeno in atto.

Vi è da notare che il massimo di intensità del vento coincide con il massimo di valore raggiunto dal gradiente e con $\mathrm{i}$ valori minimi raggiunti dalla conducibilità dell'aria, salvo il valore eccezionale del picco, dovuto alla azione di una raffica particolarmente forte e di breve intensità.

Le rapide oscillazioni della conducibilità sono probabilmente legate alla presenza delle polveri atmosferiche, la cui densità media viene rlal vento variata nel volume della colonna d'aria presa in considerazione, a causa degli apporti di elementi silicei e di altra natura presi dall'ambiente circostante (terreni agrari, maggesi, boschi).

TABELLA 2

\begin{tabular}{|c|c|c|c|c|}
\hline \multirow{2}{*}{ Ore } & \multicolumn{2}{|c|}{$\begin{array}{c}\text { Conducibiliti positiva e negativa } \\
10^{-14}\left(\mathrm{omh}^{-1} \mathrm{~m}^{-1}\right)\end{array}$} & $\begin{array}{c}\text { Gradiente di } \\
\text { potenziale } \\
V / m\end{array}$ \\
\cline { 2 - 4 } & $\lambda+\lambda_{+}$ & $\lambda-$ & \\
\hline & & & & \\
11.00 & 1.80 & 1.82 & 3.62 & 60 \\
11.30 & 1.85 & 1.90 & 3.75 & 55 \\
12.00 & 1.86 & 1.90 & 3.76 & 75 \\
12.30 & 1.90 & 1.91 & 3.81 & 70 \\
13.00 & 2.47 & 2.51 & 4.98 & 70 \\
13.30 & 2.40 & 2.51 & 4.91 & 73 \\
14.00 & 2.48 & 2.53 & 4.91 & 70 \\
14.30 & 1.66 & 1.78 & 3.44 & 92 \\
15.00 & 1.69 & 1.77 & 3.46 & 100 \\
15.30 & 1.30 & 1.36 & 2.66 & 130 \\
16.00 & 3.05 & 3.33 Picco & 6.38 & 130 \\
16.30 & 1.76 & 1.82 & 3.58 & 90 \\
17.00 & 2.87 & 3.03 & 5.90 & 88 \\
17.30 & 2.91 & 3.05 & 5.96 & 88 \\
\hline
\end{tabular}




\section{4. - Confronto CoN l'ANdamento DEI. PULViscolo atyosferico}

La fig. 6 riporta gli andamenti medi della conducibilita e della distribuzione oraria del pulviscolo atmosferico. Tale distribuzione d̀ stata rilevata durante studi sull'inquinamento atmosferico, mediante metodi ottici e ponderali, atti a mettere in evidenza la distribuzione delle polveri presenti durante tut to lanno e nelle diverse ore del giorno.

Come si può notare, le due curve presentano un notevole grarlo di antiparallelismo, che d'altra parte è stato trovato anche da diversi ricercatori $(11,3,4,7,16)$ per stazioni di ricerca site in aree urbane e non urbane.

Sul comportamento della densità delle polveri presenti nella atmosfera, ha notevole influenza la posizione dello strato di inversione

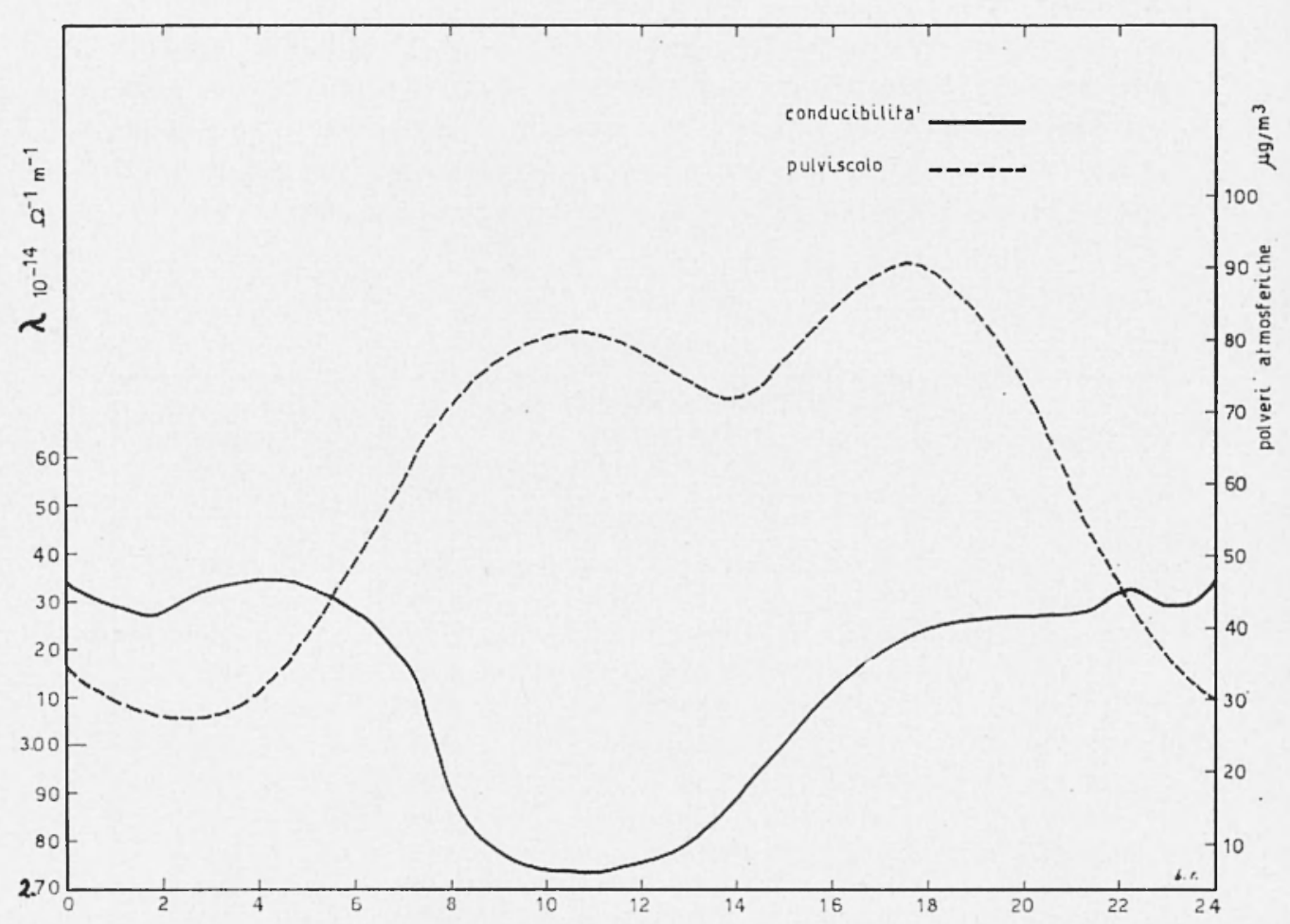

Fig. 6. - L'antiparallelismo dell'andlamento della conducibilità elettrica atmosferica e della polverositì dell'aria durante la giornata viene messo in evidenza dalla figura, sulla quale sono riportati i valori medi dei due fenomeni, rilevati nel periodo 1970-1974 (Marzo). 
termica, che nella regione si presenta ad una altezza media compresa fra i 280 ed i 450 metri sul mare, a seconda delle stagioni e delle condizioni generali.

La stazione di osservazione rimane pertanto al disotto od entro tale stato di inversione che determina, con la sua presenza, un accumulo delle sostanze inquinanti.

Il minimo di polverosità accusato verso le ore 14.00 TMIEC può attribuirsi in parte alla diminuita circolazione stradale che si nota in quell'ora ed alla diminuzione delle emissioni degli impianti domestici, ma sopra tutto alla posizione dello strato di inversione che nelle ore meridiane si innalza o si dissolve, determinando mediante correnti convettive, una diminuzione della densità delle polveri stesse.

A tale minimo corrisponde una chiara inflessione nell'andamento medio della conducibilità dell'aria, come d'altra parte ai massimi di polverosità vengono a corrispondere minimi di conducibilità e viceversa.

Premesso poi che le misure dei due parametri sono state condotte nello stesso luogo, alla stessa altezza, a conveniente distanza da sorgenti perturbanti, riteniamo si possa concludere che fra la polverosità misurata e la conducibilità esista una correlazione, che d'altra parte era preverlibile, essendo certamente le polveri portatrici di cariche elettriche, sia a causa della loro genesi, sia per fenomeni elettrostatici cui sono sottoposte nel loro moto forzato nell'aria, sia per i fenomeni di cattura di ioni da esse operato.

D'altra parte l'antiparallelismo constatato può essere giustificato dalla ridotta mobilità degli ioni, imposto dalla presenza delle polveri, mobilità che diminuisce appunto con l'aumentare della densità delle polveri stesse.

\section{5. - Conclusioni}

La conducibilità elettrica dell'aria dipende dalla presenza nell'aria stessa dei singoli gruppi di ioni.

La conducibilità è per la massima parte dovuta agli ioni piccoli, la eui mobilità ̀̀ di gran lunga superiore a quella delle altre specie di ioni che possono essere presenti.

Le nostre ricerche intese a misurare la conducibilità totale, hanno indicato un andamento stagionale ed orario del fenomeno, assolutamente coerente con gli andamenti trovati per le altre stazioni site in 
aree simili a quella dove si è operato, cioè un'area urbana di modesta estensione.

Nelle considerazioni che si possono fare sui risultati ottenuti, è necessario tenere presente che la stazione è posta sul culmine di una collina, a morlesta distanza dal mare Adriatico, le cui brezze giungono costantemente fino alla stazione di osservazione.

Gli andamenti rilevati durante fenomeni meteorologici significanti, come temporali, formazione di nebbie, venti catabatici o brezze, appaiono caratteristici, con le loro variazioni, con la modificazione repentina dei valori e dell'andamento della conducibilità, mettendo così in evidenza delle particolarità nella distribuzione e nella densità e mobilità degli ioni presenti.

Sono in particolare da mettere in evidenza le influenze esercitate dalle brezze notturne, le quali appunto stabiliscono delle singolarità locali, come il piccolo minimo da noi trovato in coincidenza con il prevalere delle brezze di monte, in certe ore durante le stagioni intermedie.

L'influenza del foehn sulla conducibilità ì in piena concordanza con quanto a suo tempo trovato riguardo al gradiente di potenziale. Quest'ultimo parametro subisce un aumento in armonia con lo stabilirsi del vento catabatico, mentre la conducibilità subisce un decremento rispetto ai valori attuali e medi della giornata, come pure rispetto ai valori merli stagionali.

La diminuzione è nettamente avvertibile, pur tenuto conto delle oscillazioni della conducibilità stessa, legate alle raffiche del vento le quali, modificando la densità e la distribuzione degli ioni presenti, stabiliscono delle oscillazioni nel valore istantaneo della conducibilità. Tali oscillazioni non sono presenti nei momenti di calma o di flusso laminare del vento.

La correlazione poi fra l'andamento della conducibilità e quello del pulviscolo atmosferico è messo in evidenza dal confronto delle due curve della distribuzione oraria dei due fenomeni.

Tale correlazione appare abbastanza stretta, almeno nella espressione dei valori merli dei due fenomeni. Le misure e le ricerche in corso permetteranno di stabilire un'espressione più evidente e precisa dell'influenza esercitata dal pulviscolo atmosferico sulla conducibilità, nell'area da noi considerata. 


\section{BIBLIOGRAFIA}

(1) Cilalmers J. A., 1967. - Atmospheric electricity. 2n ed. (cap. 7), "Pergamon Press".

(2) Citubarina E. V. - Iminitov I. M., 1967. - Electricity of the free atmosphere. Israel program for Scient. Translations.

(3) Совв W. E., 1968. - Ion losses in the Gerdien condenser intake system. "J. of App. Met. ", 7, p. $456 / 457$.

(4) Совв W. E., 1973. - Oceanic aerosol level deduced from measurements of the elec. conduc. of the Atmosph. "Journal of the Atm. Science", p. 101.

(5) Coroniti C. S. - Hugines J., 1962. - Planetary Electrodynamics. 1-2, Gordon \& Breach (Editors).

(6) Gualtieri G. - Murri A., 1969. - The vertical gradient of electroatmospheric potential at Macerata. Central East Appemnines. "Annali di Geofisica ", XXII, 1.

(7) Kaмza A. K., 1972. - Measurements of the electrical Properties of dust storms. "J. of Geoph. Res. ", 77, 30, 5856.

(8) Koenigsfeld L., 1971. - Correlations of results from atmospheric electricity measurements at four stations in Belgium. "Pageopl. ", p. 27-84.

(') Israel H., 1971. - Atmospheric electricity. Israel Prog. of Scient. Trans., 1.

(10) Jones O. C. and otilers, 1958. - Radiosonde measurement of vertical elect. field and conductivity in the lower atmosph. Recent Advances in Atm. Elec., p. 233.

(11) Mani A.-IIUddar B. B., 1972. - Studies of surface aerosol and their efjects on Atm. Elect. Parameters. "Pageoph. ", 100, VIII, p. 155.

${ }^{\left({ }^{12}\right)}$ Mulineisex R., 1965. - Report on atmospheric electricity in central Europe. 1959/1962. Problems of Atm. Elec. and Space Elec., p. 25.

$\left.{ }^{(13}\right)$ MUrri A., 1964. - Caratteristiche del gradiente verticale di potenziale e.a. a Macerata. "Geofisica e Meteor. ", 13, 1-2, Genova.

(14) MURri A., 1966. - I venti catabatici da ovest nell'A ppennino centrale. "Rendiconti Osser. Meteor. di Macerata", III, 2.

$\left.{ }^{15}\right)$ Murri A.-Fusari R.-Scuterini C., 1973. - The behaviour of some electro-atmospheric and meteor. parameters during the partial eclipse of June 30th 1937. "Rendiconti Osserv. Meteor. di Macerata ", III, 4.

${ }^{(16)}$ Runnke I. H., 1966. - Visibility and small ion density. "J. of Geoph. Res. ", 71, 18, p. 4235.

(17) SAGALYN R. C., 1958. - Significance of ratio of the polar conductivity. Recent Advances in Atm. Elect., p. 235.

${ }^{(18)}$ Srivastava G. P. and others, 1972. - Electrical conductivity and gradient measurement in the free atmosphere over India. "Pagcoph.", 100, VIII, p. 81.

(19) Vercelli F., 1952. - L'aria. UTE'T, p. 489 e seg. 